STUDI

FRANCESI

\section{Studi Francesi}

Rivista quadrimestrale fondata da Franco Simone

190 (LXIV | I) | 2020

Varia - fasc. I - gennaio-aprile 2020

\title{
Lectures et lecteurs de Stendhal, dir. M.-R. CORREDOR, B. DIDIER et $\mathrm{H}$. de JACQUELOT
}

\section{Michel Arrous}

\section{(2) OpenEdition \\ Journals}

Édition électronique

URL : https://journals.openedition.org/studifrancesi/22816

DOI : 10.4000/studifrancesi.22816

ISSN : 2427-5856

\section{Éditeur}

Rosenberg \& Sellier

\section{Édition imprimée}

Date de publication : 1 avril 2020

Pagination : 188-190

ISSN : 0039-2944

\section{Référence électronique}

Michel Arrous, "Lectures et lecteurs de Stendhal, dir. M.-R. Corredor, B. Didier et H. de Jacauelot », Studi Francesi [En ligne], 190 (LXIV | I) | 2020, mis en ligne le 01 mai 2020, consulté le 03 août 2021. URL : http://journals.openedition.org/studifrancesi/22816 ; DOI : https://doi.org/10.4000/studifrancesi. 22816

Ce document a été généré automatiquement le 3 août 2021.

\section{(†)

Studi Francesi è distribuita con Licenza Creative Commons Attribuzione - Non commerciale - Non opere derivate 4.0 Internazionale. 


\title{
Lectures et lecteurs de Stendhal, dir. M.-R. CORREDOR, B. DIDIER et H. de JACQUELOT
}

\author{
Michel Arrous
}

\section{RÉFÉRENCE}

Lectures et lecteurs de Stendhal, dir. M.-R. CORREDOR, B. DIDIER et H. de JACQUELOT, Paris, Champion, 2019, $343 \mathrm{pp}$.

1 Témoignage d'échanges et de confrontations dans le cadre d'un séminaire qui s'est tenu de 2004 à 2011 à Grenoble, Rome et Pise, ce riche volume a un double thème: «Lectures de Stendhal» - essentiellement Stendhal lecteur du XVIII ${ }^{\mathrm{e}}$ siècle - et «Lecteurs de Stendhal européens et modernes», soit quelques incursions du côté de Suarès, Valéry, Ortega y Gasset, Gadda, Sciascia, Calvino, et Zamiatine.

2 Stendhal moliéresque (pp. 11-29), tel est le point de départ choisi par Francesco SPANDRI, aussi bon connaisseur de la monomanie théâtrale de Balzac que du théâtre avorté de Beyle. Ce dernier a voulu perfectionner ou dépasser Molière, être le «Molière tragique». Notre aspirant dramaturge, qui reprend la thèse de Rousseau, procède à une lecture critique du Misanthrope, sujet «raté» parce que le protagoniste connaît insuffisamment le malheur. De ses lectures raisonnées de 1802-1804 et 1813, Beyle conclut que doit primer le combat des passions et que le ridicule naît du désappointement de l'être passionné. C'est son «désordre» que le poète devra «comiquer». Revenant à la question de la réception générale des écrivains du XVIII ${ }^{\mathrm{e}}$ siècle au début du XIX ${ }^{\mathrm{e}}$, Béatrice DIDIER s'est livrée à une enquête pour préciser la position de Stendhal (Dans le sillage des moralistes et de Montesquieu, pp. 31-48). Conclusions provisoires: pour Stendhal, le XVIII ${ }^{\mathrm{e}}$, siècle auquel il est resté fidèle, c'est un modèle de style; quand il se croit dramaturge, il étudie le théâtre de Voltaire, les théories de Diderot et les moralistes, dans une préoccupation morale ou pour y chercher des vérités personnelles, mais aussi avec le 
souci de la forme quand il s'intéresse à la maxime, au portrait ou à l'anecdote. Le souci du style, on le retrouve quand il lit Montesquieu - il l'a lu tout au long de sa vie - en qui il a vu un maître du style concis, tout à l'opposé de la prolixité et du vague des Italiens, ou du style sensible de Rousseau. Ce souci se double d'une réflexion sur la politique de Montesquieu qu'il discute parfois. Et Voltaire? Sylvain MENANT (L'image de Voltaire en France au temps de Stendhal, pp. 49-54) rappelle la situation du philosophe dans l'opinion au début du XIX ${ }^{e}$ siècle: sous la Restauration, Voltaire occupe une place centrale, son œuvre est un immense succès de librairie. Le Voltaire que fréquente Stendhal, c'est celui du théâtre et des satires. L'image du philosophe est alors essentiellement polémique; dans le cas de Stendhal, elle s'est très tôt figée en quelques traits qui résument le personnage et l'œuvre, depuis le temps où le jeune Henry Brulard révérait chez son grand-père le buste de Voltaire et prélevait quelques volumes des Euvres complètes dans la bibliothèque de son père. Ce sont ces premières explorations que retrace Béatrice DIDIER (Toucher le buste de Voltaire, pp. 55-60). Sous un angle différent et fort original, Laurence MACÉ examine les rapports entre les deux auteurs (De quoi Voltaire est-il le nom? De la présence du philosophe et de l'homme de théâtre dans l'Italie de Stendhal, pp. 61-79). La présence majoritaire de Voltaire dans les récits de voyage en Italie de Stendhal a-t-elle un rapport avec la présence attestée de Voltaire dans l'Italie de l'époque? Il se pourrait que l'héritage voltairien, surtout la passion pour le théâtre et la détestation des bigots, date de l'expérience italienne des années 1800-1801. Après cette date, on ne cessa pas pour autant de lire Voltaire, malgré la censure autrichienne et romaine, mais, si l'on en croit Stendhal, les Italiens, victimes d'une longue soumission intellectuelle, n'adhéraient en rien aux idées esthétiques et philosophiques de Voltaire, encore moins à l'«esprit français» qu'il représentait. Cela dit, on n'oubliera pas que Rossini a transformé les grandes tragédies voltairiennes en opéra (Tancredi, Semiramide). L'attitude de Stendhal à l'égard de Voltaire est complexe: d'un côté, la figure positive de l'écrivain engagé, de l'autre, le dramaturge incarnant le néoclassicisme littéraire (auquel on doit cependant l'introduction de Shakespeare en France), que Stendhal a abondamment pratiqué et dont l'œuvre a joué un rôle déterminant dans son engagement romantique. Agathe NOVAK-LECHEVALIER passe en revue ses jugements sur les tragédies et les essais critiques de Voltaire sur le théâtre (Stendhal, lecteur du théâtre de Voltaire, pp. 71-85). Quoiqu'il en recommande la lecture à sa sœur, il n'est à ses yeux que le second de Racine, une pâle copie de Corneille et de Racine. Voltaire échoue parce qu'il méconnaît le principe des passions et parce qu'il est plus épique que dramatique. Voltaire est bien la quintessence de l'«esprit français», mais il n'a aucun «sentiment des arts». La critique vise aussi la comédie où Voltaire échoue à faire rire et, fait plus grave, Voltaire n'est qu'un plagiaire (Zaïre adaptée d'Othello), d'ailleurs plagié par d'innombrables disciples (Ancelot, Jouy, Soumet). Ce qui sauve son œuvre critique, c'est sa puissance subversive dans sa lutte contre la tyrannie et le fanatisme. Stendhal n'a pas été sensible aux innovations dramaturgiques de Voltaire. Donc un intérêt ambigu et complexe, et une présence qu'on constate jusque dans certains projets comme L'Orgueilleux (ou Voltaire à Ferney), Les Deux hommes, et Letellier où sévit un anti-voltairien. Jean SGARD (Stendhal et la "complainte noble» de Prévost, pp. 87-94) et Jean-Jacques LABIA (Prévost, "Le Doyen de Killerine" ou «l'amour tel qu'il est dans un roman moderne», pp. 95-111) consacrent leurs contributions à la connaissance de Prévost découvert lors d'une lecture subreptice des Mémoires d'un homme de qualité. La plupart des très nombreuses traces de lecture disséminées dans l'œuvre de Stendhal illustrent le thème de la «grande passion» et le goût du grand romanesque («chaud 
comme un roman de Prévost», dira Stendhal à propos de la Sainte-Pétronille du Guerchin). Le génie de Prévost se révèle dans la peinture de la "violente maladie», qu'on pense à la foisonnante saga familiale racontée dans Le Doyen de Killerine, ou, pour le même roman, à l'enthousiasme de Lisio Visconti dans De l'amour. Stendhal, sensible au style soutenu, voit dans les romans de Prévost des modèles aussi éloquents que $L a$ Princesse de Clèves, La Nouvelle Héloïse ou les Lettres d'une religieuse portugaise. Stendhal l'a lu très tôt, de même pour Diderot. À partir des inventaires de sa bibliothèque, B. DIDIER essaie de déterminer les textes que Stendhal a pratiqués, les œuvres qui l'ont le plus marqué et l'époque où il les a lues (Les volumes bleus de "l'Encyclopédie" et Diderot leur maître d'œuvre, pp.113-128). Bien avant la découverte de Jacques le Fataliste, des Entretiens et des Salons, l'enfant Beyle avait ouvert l'Encyclopédie dont Stendhal dira beaucoup plus tard que c'est «l'ouvrage le plus utile jamais publié». Stendhal a beaucoup pratiqué l'«athée furibond» qu'il trouve «un peu emphatique», aussi lui préfère-t-il Helvétius. Ce jugement sur l'emphase de Diderot, Jacques LAMBERT le nuance dans «Dépecer l'hippogriffe»: remarques sur Stendhal lecteur de Diderot (pp. 129-142). De l'emphase, le Touriste dira qu'elle est «cousine germaine de l'hypocrisie des prêtres»; elle est l'antagoniste de l'énergie, et Stendhal la retrouve dans l'Essai sur la peinture, dans les pages sur l'expression, et bien sûr dans ce que Diderot dit de Greuze. En sont exempts Jacques le fataliste et les pages du Salon de 1767 où Diderot privilégie l'esquisse comme «saisie quasi immédiate de l'essentiel bien "senti"». Présentée par B. DIDIER (Rousseau multiple, pp.143-145), l'«immense et complexe» relation de Stendhal à Rousseau a suscité trois interventions. D'abord une analyse barthésienne du rapport à la fois poétique, rhétorique et stylistique qu'entretiennent Les Confessions et le Brulard, ce que Damien MAZARÉ nomme l'intermédialité dans la modélisation de la pratique autobiographique (De la chambre noire à la mante noire: ce que la lecture de Rousseau nous apprend de la représentation stendhalienne, pp.147-162). Plutôt que d'interroger l'ensemble de l'œuvre, Cécile MEYNARD a examiné La présence de Rousseau dans les journaux et papiers de Stendhal (pp.163-178). De nombreux renvois à Rousseau, pas toujours suivis d'une analyse (à propos, par exemple, de la manipulation du lecteur par le style) attestent que Rousseau est toujours une référence ou un repère dans son autoformation, y compris quand il exprime ses doutes sur le penseur. Quant aux Confessions, elles servent à la fois de modèle et de repoussoir, à l'époque de la Vie de Henry Brulard, quand Rousseau «reste épisodiquement présent dans sa vie, mais d'une façon distanciée». Dans l'héritage culturel de Stendhal et à l'origine de la singularité du Beyliste, il y a aussi l'auteur du Contrat social. Même si le disciple d'Helvétius et des Idéologues a beaucoup moins pratiqué l'ouvrage de 1762 que La Nouvelle Héloïse et Les Confessions, on peut voir dans sa lecture une résistance à Rousseau, d'autant plus forte qu'elle s'appuie sur la sévère critique des préjugés optimistes du «bon sauvage» trouvée chez Volney (Michel ARRous, Stendhal et les «utopies impossibles» de Rousseau, pp. 179-188). À la veille de son premier séjour à Rome en octobre 1811, quels livres Stendhal avait-il lus? Des récits de voyage, le roman de Mme de Staël, Corneille, Tite-Live - un vœu pieux -, d'autres lectures académiques et des références classiques, ainsi Beyle construit «sa propre image spécifique de Rome» dont Letizia NORCI CAGIANO dit qu'elle sera «marquée par une profonde désillusion» (Stendhal et la «speme del viaggio a Roma», pp. 189-197). Avec la co-éditrice des Idées italiennes sur quelques tableaux célèbres (1840), on verra dans Stendhal un véritable lecteur de métier. Hélène de JACQUELot le montre à l'œuvre dans L'horizon de lecture des "Idées italiennes" (pp. 199-217). Au cours des dernières relectures, 
Stendhal, constant dans sa pratique des «manipulations citationnelles», emprunte aux historiens de la peinture et aux voyageurs.

Moins fournie, la deuxième partie, «Lecteurs de Stendhal européens et modernes», aborde quelques aspects de l'héritage littéraire de Stendhal. Paola CATTANi a eu l'excellente idée de traiter un sujet méconnu (André Suarès lecteur de Stendhal. La querelle sur le style, pp. 221-236). Le long article «D'après Stendhal» (1914) n'est pas seulement la défense d'un style souvent contesté et discrédité, c'est aussi la défense polémique d'une conception de la littérature, contre Brunetière et Faguet, contre Barrès et contre l'assimilation entre Nietzsche et Stendhal, que Suarès reprendra en la renforçant dans «Stendhal, Verlaine, Gérard de Nerval et autres gueux» et le Voyage du condottière. Plus attendu: Valéry, cas fameux de la critique stendhalienne sur lequel revient Antonietta SANNA, à partir des Cahiers où d'innombrables notes concernent Stendhal, moins comme romancier que comme scrutateur (Le théâtre intime de Stendhal. Paul Valéry lecteur, pp. 237-244). Peu connu en France, le métaphysicien Ortega y Gasset a publié en 1926 «L'amour chez Stendhal» dont Marie-Rose CORREDOR donne une analyse suivie (Ortega $y$ Gasset lecteur de "De l'Amour", pp. 245-254). Le philosophe espagnol fait du traité une lecture réductrice et même faussée quand il analyse la "cristallisation». Avec la collaboration de Tatiana NIKITINA, Alexandre STROEV étudie un projet d'adaptation de Vanina Vanini qui transforme jusqu'à la dénaturer la nouvelle de Stendhal (Evgueni Zamiatine adaptateur de Stendhal: le scénario inédit de "La princesse Vanina" (1936), pp. 255-261). Les premières tentatives romanesques de Gadda, lecteur attentif du Rouge et sensible à l'ironie stendhalienne, sont bien plus intéressantes que ce scénario imbibé d'«humanisme prolétarien». Alberto GoDiol le prouve sans peine avec Mensonges romantiques et vérités romanesques. Gadda lecteur de Stendhal (pp. 263-279): le futur auteur de L'Affreux Pastis de la rue des Merles adopte une technique du point de vue proche de celle de Stendhal et, comme lui, se préoccupe du «lien entre société démocratique et développement des pulsions égotistes». À ce cas de stendhalisme, il faut ajouter celui de «l'homme à la loupe», Leonardo Sciascia qui, dans L'Adorable Stendhal, «a professé la passion stendhalienne la plus bigarrée», et plus encore dans Stendhal et la Sicile (1984) où il démontre que notre voyageur ne la visita jamais. Dans cette passion, Domenico SCARPA (Esprits stendhaliens. Sciascia et le désir, pp. 281-290) voit «une cleptomanie au second degré» grâce à laquelle Sciascia s'évade de l'actualité italienne (la mafia, la loge P2, les Brigate Rosse, les néo-fascistes, etc.). Pour Jean-Jacques LABIA, il en va de même chez Calvino: dans son monde décalé, face au «classique» Stendhal, il s'interroge sur la poétique de l'autobiographie (Stendhal-Calvino: une poétique du soupçon, pp. 291-305). Chantal MASSOL donne un dernier coup de sonde (Le «compagnon familier»: Beyle lu par W.G. Sebald, pp.307-331). Le spécialiste de littérature allemande revendiquait une «relation privilégiée» avec Flaubert, Balzac et Stendhal. Dans son recueil Vertiges (1990) figure une fiction biographique où apparaît un Stendhal mélancolique saisi dans un réseau de traces et de signes. L'héritage littéraire du «cosmopolite» Stendhal est largement européen. 